\title{
Optimal Broadcast Channel for Data Dissemination in Mobile Database Environment
}

\author{
Agustinus Borgy Waluyo ${ }^{1}$, Bala Srinivasan ${ }^{1}$, and David Taniar $^{2}$ \\ ${ }^{1}$ School of Computer Science and Software Engineering, \\ Monash University, Australia \\ \{Agustinus. Borgy.Waluyo, Bala.Srinivasan\} a infotech.monash.edu . au \\ ${ }^{2}$ School of Business Systems, \\ Monash University, Australia \\ David.Taniar@infotech.monash.edu.au
}

\begin{abstract}
The increase number of mobile users in wireless environment affects query access time substantially. To minimise the query access time, one possible way is to employ data broadcasting strategy. In this paper, we propose cost models for both query access time over broadcast channel and on-demand channel. We examine the cost models to find optimum number of broadcast items in a channel while utilising query access time over on-demand channel as a threshold point. The optimum number indicates a point to split the broadcast cycle and allocate the data items in the new channel or else the on-demand channel outperforms the broadcast channel. The cost model involves several factors that dynamically change the optimum number of broadcast items like request arrival rate, service rate, size of data item, size of request, and bandwidth. Simulation model is developed to verify the performance of the cost model. This paper focuses on request that returns a single data item.
\end{abstract}

\section{Introduction}

The invention of wireless technology has created a new era of computing application. Nowadays, people are no longer attached to a stationary machine to do their work, with wireless application they are enabled to conduct their business anywhere and anytime using portable size wireless computer powered by battery. These portable computers communicate with central stationary server via wireless channel. This technology is known as mobile computing $[2,3,6]$. Mobile computing has its own database management system (DBMS) that provides the same function as in traditional databases, which is called mobile databases. Mobile databases face a number of limitations particularly power, storage and bandwidth capacity. In regard to power capacity, it has been investigated that the life expectancy of a battery is anticipated to increase only $20 \%$ for every 10 years [11]. Consequently, the need to use power efficiently and effectively is a crucial issue. In a mobile environment, most applications involve read operations rather than write operations [5]. This paper concerns with broadcast strategy to optimize the read operation or referred as query operations in mobile databases. 
Broadcast strategy refers to periodically broadcast database items to clients through one or more broadcast channels. Mobile clients filter their desired data on the fly. This strategy is known as an effective way to disseminate database information to a large set of mobile clients. The query performance using this strategy is independent from the number and frequency of the query, or known as scalable paradigm. However, the challenge in broadcast strategy is to maintain the query performance of the client to obtain information from the channel.

It is the aim of this paper to optimize the query access time of accessing data from broadcast channel. The query access time over on-demand channel is used as a threshold point to determine the optimum number of database items to be broadcast in a channel. Once, the optimum number is located, the number of broadcast channel should be increased. Subsequently, the length of broadcast items is split, and broadcast over multiple channels. With this strategy, the access time of mobile users for accessing data items over broadcast channel is kept considerably low.

To achieve our objective, we propose cost models for both on-demand and broadcast channel. Subsequently, we use simulation model to verify the results of our cost models in finding the optimum number of broadcast items. In this paper, we focus on request that returns single data items.

The rests of the section in this paper are organised as follows. Section 2 describes the background of mobile databases, and section 3 contains the related work of the proposed technique. It is then followed by the description of the optimal broadcast channel, cost model for both broadcast and on-demand channel, and its application in section 4. Section 5 introduces parameters of concern of the optimal broadcast channel, which includes performance results of cost models and simulation models to determine optimal broadcast channel. Finally, section 6 concludes the paper.

\section{Background}

In general, each mobile user communicates with a Mobile Base Station (MBS) to carry out any activities such as transaction and information retrieval. MBS has a wireless interface to establish communication with mobile client and it serves a large number of mobile users in a specific region called cell. Mobile units or mobile clients in each cell can either connect to the network via wireless radio or wireless Local Area Network (LAN). Wireless radio bandwidth has asymmetric communication behavior in which the bandwidth for uplink communication is smaller than downlink communication [7]. Uplink bandwidth is applicable when mobile clients send a request or query to the server, while downlink bandwidth is from the server back to the clients.

With the increase number of mobile users in a cell, the required number of data items to be broadcast also increases accordingly. This situation may cause some mobile clients to wait for a substantial amount of time before receiving desired data item. Consequently, the advantages of broadcast strategy will be eliminated. On the other hand, when the channel contains too few data items, a high percentage of mobile clients waste the time by listening to the channel until they find out that the desired data is not broadcast. Alternatively, they send a request via point-to-point channel or on-demand channel to the server. The server processes the query and sends 
the result back to the client. The last scenario severely affects the power consumption of mobile clients, as it involves queuing in the server.

Figure 1 illustrates the two mechanisms of mobile clients to obtain the desired data, first mechanism is via on-demand channel, and the other is via broadcast channel. It is sometimes called pull-based, and push-based approach respectively. In this paper, the term mobile client, mobile computer, mobile device, mobile user and client are used interchangeably and the server refers to MBS.

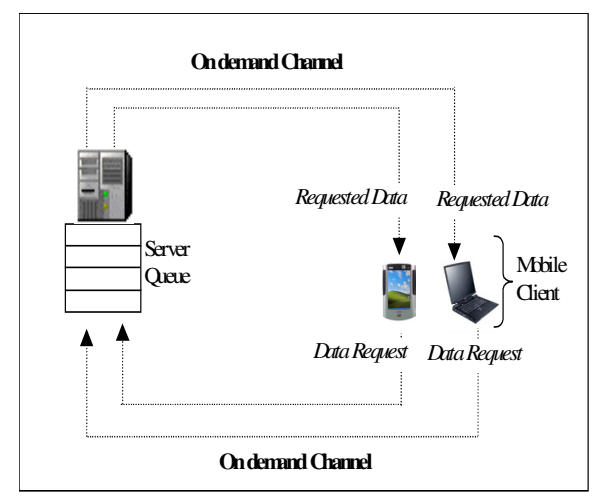

(a) On demand Channel

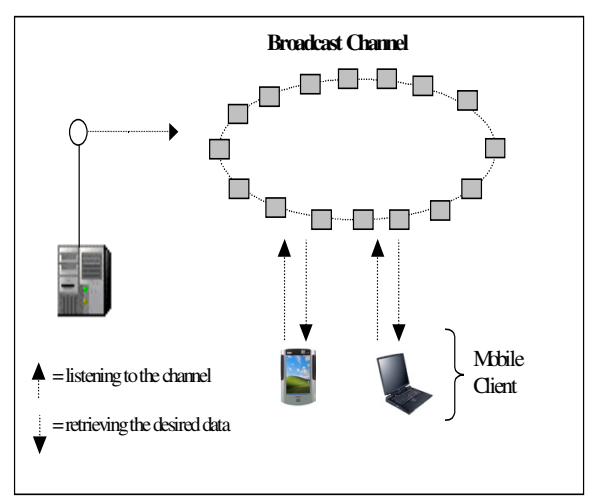

(b) Broadcast Channel

Fig. 1. The architecture of on-demand channel and broadcast channel

\section{Related Work}

Strategies to broadcast data through wireless channel have been introduced in the past few years $[1,8,9,12]$. Broadcast strategy is known to be effective when involving a large number of users in the cell, which corresponds to a high frequency of requests.

[4] has introduced the hybrid and dynamic channel allocation method, which manipulates both the on-demand channels and broadcast channels. The hybrid allocation method provides a solution by determining the number of data to be served from on-demand channel and broadcast channel to enhance the query performance. Dynamic hybrid method is the improvement of the hybrid method, which changes the number of channel allocated for on-demand mode and broadcast mode dynamically by considering the load of the server and request access pattern. However, the access overhead of on-demand channels only considers the waiting time of connection to the server. Several factors that should be taken into account include transmission cost of the query from the mobile client to the server, processing cost in the server and the cost to send the query response back to the mobile client. The cost to transmit the query to server is even worse when the query requires related data items that belong to a number of entity types due to the uplink bandwidth limitation. Furthermore, the frequency or the rate of the query initiated affects the performance of the on-demand channel.

Our optimal broadcast channel tries to eliminate the consequence of having a massive set of data to be broadcast. The strategy is used to determine the optimum number of broadcast items in a channel so that the average access time is kept minimum [13]. 


\section{Optimal Broadcast Channel}

Optimal broadcast channel is designed to find out optimum number of broadcast items in a broadcast channel. Having known the optimum number of broadcast items in a channel enables us to determine the optimum number of channel required to broadcast a certain number of data items. In this paper, the query performance is measured by the average access time of broadcast channel. Access time is defined as the amount of time or waiting time needed from the time a request is initiated until data item of interest is received. In general, the data items are broadcast over a single channel with underlying reason that it provides the same capacity (bit rate/bandwidth) as multiple channels, and it is used to avoid problems such data broadcast organization and allocation while having more than one channel [7]. Nevertheless, the use of single channel will show its limitation when there is a vast number of a data item to be broadcast.

The proposed strategy is used to split the length of the broadcast cycle when the number of broadcast items reaches an optimum point. Figure 2 illustrates a situation when the optimum number of items in a channel is known.

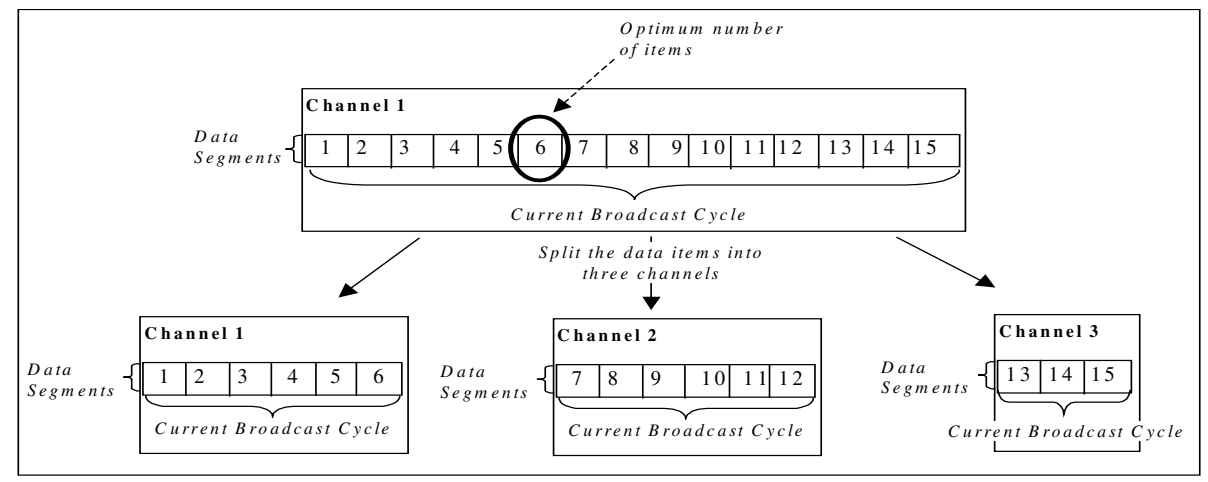

Fig. 2. Optimal Broadcast Channel

The allocation of broadcast cycle into an optimal number of broadcast channels will eliminate the chance of long delay before obtaining the desired data items. As mobile clients are able to listen to different channels simultaneously, they can determine which channel contains the required data items. Once it has been found, they may wait to retrieve the data items in the corresponding channel. Since the length of the broadcast cycle is optimal, the waiting time will be considerably short.

To find the optimum number of broadcast items, we develop cost models to calculate average access time of broadcast and on-demand channel. In this context, mobile client can only initiate a single request at a time, the next request has to wait until the first request has been completely responded.

\subsection{Cost Model for Broadcast Channel}

To calculate the average access time for retrieving data $\left(T_{B}\right)$ using broadcast channel, we considers the following scenario: 


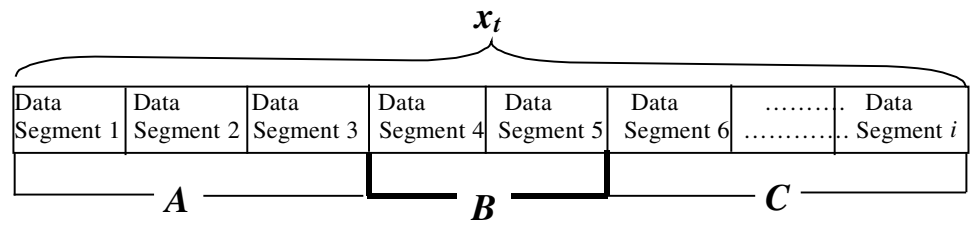

Fig. 3. Broadcast Cycle Partitioned Area

In figure 3 , the broadcast cycle is partitioned into three areas: area $A$ contains data segments preceding data segments in area $B$, area $B$ contains the number of desired data segments, and area $C$ includes the rest of data segments. $x_{t}$ is the total number of broadcast data that makes up of $A+B+C$. There are three different scenarios when mobile client probe into one of these area.

Probe A: When mobile client probes into area $A$, the total access time is given:

$$
\frac{\sum_{i=0}^{A-1}(A-i+B) \times s}{b_{s}} \text { or similarly } \frac{[A \times(A+2 B+1)] \times s}{2 \times b_{s}}
$$

Probe B: When mobile client probes into area $B$, then the total access time is equal to the total length of broadcast cycle $(A+B+C)$ :

$$
\frac{(A+B+C) \times B \times s}{b_{s}}
$$

Probe C: When mobile client probes into area $C$, the total access time can be calculated from:

$$
\frac{\sum_{i=0}^{C-1}(C-i+A+B) \times s}{b_{s}} \text { or equally } \frac{C \times(2 A+2 B+C+1) \times s}{2 \times b_{s}}
$$

We need to calculate the average access time from equation (1), (2), and (3) as follows:

$$
\frac{[(A \times(A+2 B+1))+(2 B \times(A+B+C))+(C \times(2 A+2 B+C+1))] \times s}{2 \times x_{t} \times b_{s}}
$$

Equation (4) can be rewritten as:

$$
T_{B} \approx \frac{\left[\left(x_{t}-B\right)^{2}+\left(2 \times B \times x_{t}\right)+\left(2 \times B \times\left(x_{t}-B\right)\right)+\left(x_{t}-B\right)\right] \times s}{2 \times x_{t} \times b_{s}}
$$

$s$ corresponds to the size of data item, we consider the size is uniform. The downlink bandwidth is denoted by $b_{s}$. 


\subsection{Cost Model for On-Demand Channel}

As mentioned earlier, mobile clients can send their request to be processed via pointto-point or on-demand channel. Thus, the average access time of on-demand channel $\left(T_{D}\right)$ is made for comparison. It is used as a threshold point to determine the optimum number of database items to be broadcast in a channel. To calculate the average access time of on-demand channel, we classify the cost model into the following scenarios:

\section{a. Pre-determined Number of Request}

In this scenario, the total number of request has been known. The average access time can be calculated using the following cost model:

$$
T_{D} \approx \frac{r}{b_{r}}+\frac{\sum_{i=1}^{n} i \times \frac{1}{\mu}}{n}+\frac{s}{b_{s}}
$$

The cost model to calculate the access time of on-demand channel in equation (6) is based on the architecture that is depicted in figure 1a. The access time of ondemand channel comprises of the time needed to transmit a request to the server, process the request that involves the time spent in the server queue and retrieve the relevant data, and send the result back to the client. The transmission of request to the server and send the requested data item back to mobile client are affected by uplink bandwidth, $b_{r}$ and downlink bandwidth, $b_{s}$. Size of a request and the size of requested data item are indicated by $r$ and $s$ respectively, $n$ denotes the total number of request, $i$ reads the request number and the server service rate is given by $\mu$. It is assumed the request size is uniform, and each request returns a same size of data item. However, in real situation the number of requests is hardly known. Thus, we consider arrival rate of request, which is described in the subsequent strategy.

\section{b. Request Arrival Rate}

We replace determined number of request with the arrival rate of request $(\lambda)$. Furthermore, we specify the cost model based on the load of the server $(\rho)$. Thus, the average access time of on-demand channel $\left(T_{D}\right)$ is now calculated using the following cost model:

If the server is at light load $\rho=\left(\frac{\lambda}{\mu} \leq 1\right)$, then:

$$
T_{D} \approx\left(\frac{r}{b_{r}}+\frac{1}{\mu}+\frac{s}{b_{s}}\right)
$$

Else if the server at heavy load $\rho=\left(\frac{\lambda}{\mu}>1\right)$, then:

$$
T_{D} \approx\left(\frac{r}{b_{r}}\right)+\frac{\sum_{i=1}^{q \max }\left(i \times \frac{1}{\mu}\right)-\left[(i-1) \times \frac{1}{\lambda}\right]}{q \max }+\left(\frac{s}{b_{s}}\right)
$$

The average time in server queue and average processing time by the server are dynamically changed depending on the arrival rate of request $(\lambda)$ and service rate $(\mu)$. The maximum number of request in the server queue is defined by $q$ max. 
Table 1. Parameters of Concern

\begin{tabular}{|c|c|c|}
\hline Parameter & Description & Initial Value \\
\hline \multicolumn{3}{|c|}{ Broadcast channel } \\
\hline$x_{t}$ & Total number of Broadcast Items & 250 \\
\hline$B$ & Number of relevant data item & 1 \\
\hline \multicolumn{3}{|c|}{ On-demand channel } \\
\hline$n$ & Number of request & 100 \\
\hline$b_{r}$ & Uplink Bandwidth & 192 bytes \\
\hline$r$ & Size of request & 50 bytes \\
\hline$\mu$ & Server Service Rate & $\begin{array}{c}2 \text { request per } \\
\text { second }\end{array}$ \\
\hline \multicolumn{3}{|c|}{ Broadcast and On-demand channel } \\
\hline$b_{s}$ & Downlink Bandwidth & 2000 bytes \\
\hline$s$ & Size of each data item & 500 bytes \\
\hline
\end{tabular}

\section{Performance Evaluation}

In this section, we introduce two cases, and employ the cost model to determine the optimum number of broadcast data items. We also develop some simulation models to compare with our analytical results. The simulation is carried out using a simulation package Planimate, animated planning platforms [10].

The simulation environment is set to apply random delays for both arrival rate and service rate with given average value. We run the simulation process for three times, and derive the average result accordingly.

Case 1: To find the optimum number of broadcast items based on set of parameters in table 1 .

As shown in figure 4, we try to find the cross line of on-demand channel and broadcast channel. The average access time of on-demand channel is the threshold point, which appears in a straight line since the number of broadcast items does not affect its value. Our analytical results stay closely with the simulation results. The intersection point indicates the optimum number of broadcast items.

Case 2: Introducing a new parameter, arrival rate of request $(\lambda)$. Given the set of parameters in case 1 , we specify one request arrival rate per second, $\lambda=1$, to obtain server utilization $\rho \leq 1$, and three request per second, $\lambda=3$, to get $\rho>1$.

As shown in figure 5 (a), the on-demand channel performs well when the traffic request is low. The broadcast channel seems to have to allocate its data items in every 5-10 data items to a new channel to keep up with the performance of on-demand channel. However, this is not a good way since the number of channels may be excessive and may cause some overhead by listening to too many channels. Thus, ondemand channel in this case is a better way. Figure 5(b) illustrates the optimum number of broadcast items when the on-demand channel is at heavy load.

From table 3, we can see the optimum number of broadcast items derived from our analytical and simulation results on the two cases. Our analytical calculation performs very well as compared to the simulation, especially for case 1, and case 2 (server utilization > 1) with $9 \%$ and $4 \%$ error rate respectively. As for case 2 (server 


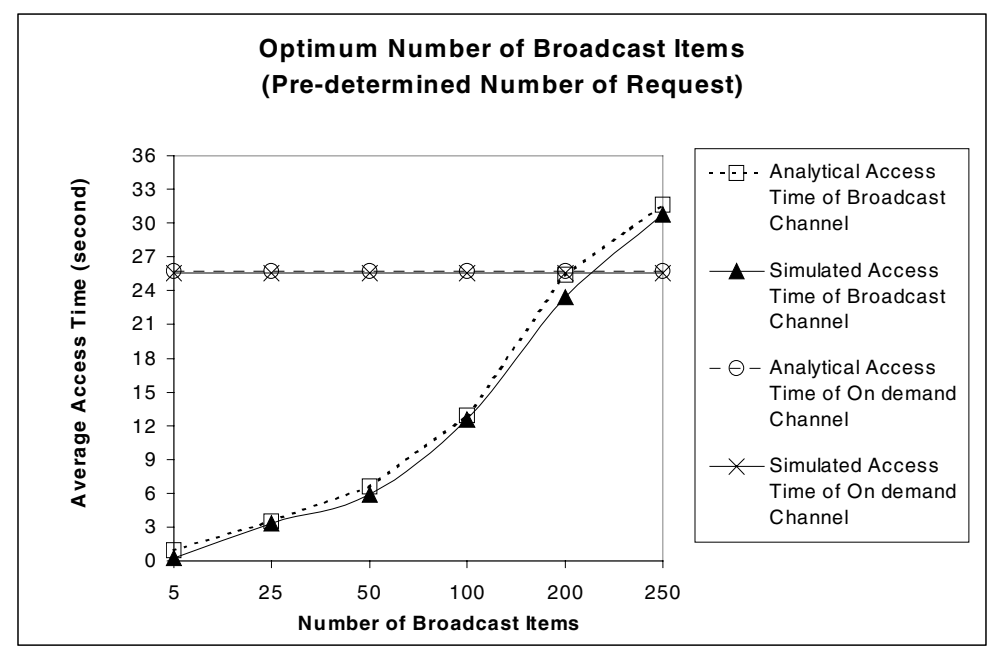

Fig. 4. Optimal Number of Broadcast Items with pre-determined number of request

Table 2. Average Access Time of On-demand and Broadcast channel

\begin{tabular}{|c|c|}
\hline \multicolumn{2}{|c|}{ On-Demand Channel } \\
\hline $\begin{array}{c}\text { Analytical Average } \\
\text { Access Time (sec) }\end{array}$ & $\begin{array}{c}\text { Simulated Average } \\
\text { Access Time (sec) }\end{array}$ \\
\hline 25.760 & 25.55 \\
\hline
\end{tabular}

\begin{tabular}{|c|c|c|}
\hline \multicolumn{3}{|c|}{ Broadcast Channel } \\
\hline $\begin{array}{c}\text { Number of } \\
\text { Broadcast Items }\end{array}$ & $\begin{array}{c}\text { Analytical } \\
\text { Average Access } \\
\text { Time (sec) }\end{array}$ & $\begin{array}{c}\text { Simulated } \\
\text { Average Access } \\
\text { Time (sec) }\end{array}$ \\
\hline 5 & 0.95 & 0.25 \\
\hline 25 & 3.49 & 3.375 \\
\hline 50 & 6.62 & 5.93 \\
\hline 100 & 12.8725 & 12.57 \\
\hline 150 & 25.37375 & 23.41 \\
\hline 200 & 31.624 & 30.75 \\
\hline
\end{tabular}

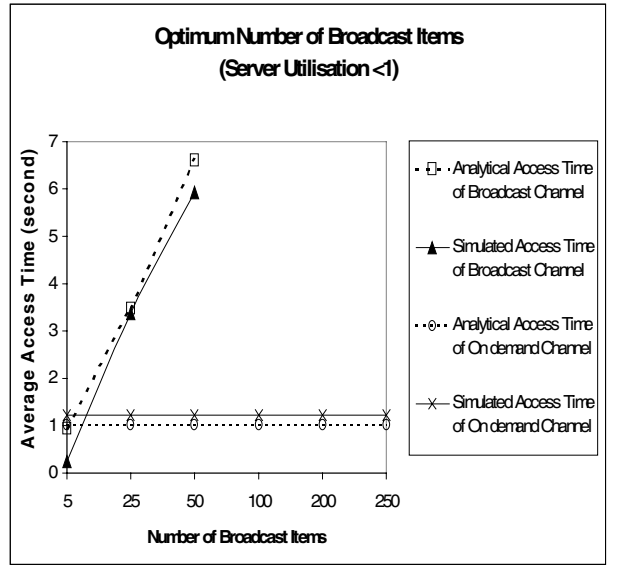

(a) Server Uilisation $<1$

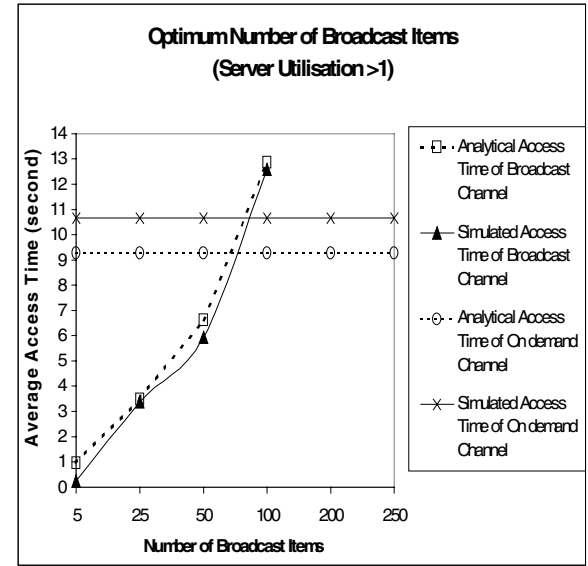

(b) Server Uilisation $>1$

Fig. 5. Optimal Number of Broadcast Items with $\rho<1$ and $\rho>1$ 
Table 3. Simulation and Analytical Performance in Determining Optimum Broadcast Items

\begin{tabular}{|c|c|c|c|c|c|}
\hline \multicolumn{6}{|c|}{ Optimum Number of Broadcast Items in a Channel } \\
\hline \multirow{2}{|c|}{ Case } & $\begin{array}{c}\text { Pre-determine } \\
\text { Number of } \\
\text { Requests }\end{array}$ & 204 & 225 & 21 & $\begin{array}{c}\text { Error } \\
\text { Rate }\end{array}$ \\
\hline 2 Case & $\begin{array}{c}\text { Server } \\
\text { Utilisation }<1\end{array}$ & 5 & 9 & 4 & 0.09 \\
\hline \multicolumn{2}{|c|}{$\begin{array}{c}\text { Server } \\
\text { Utilisation }>1\end{array}$} & 71 & 74 & 3 & 0.44 \\
\hline
\end{tabular}

utilization $<1$ ), we presume that since the value is too small, the random delays in simulation parameters cause a greater affect towards optimal number of broadcast items.

In general our analytical calculation presents a less value than the simulation. Our cost models to find an optimum number of broadcast items are considered close to accurate. Subsequently, having known the optimum number of items in a broadcast channel, we can decide how many channels are required to broadcast a certain amount of data items. Thus, the average access time of broadcast channel is kept minimum.

\section{Conclusions and Future Work}

Data dissemination or known as data broadcasting strategy is an effective way to keep up with number of clients in a cell and their frequency of requests. Mobile clients are enabled to retrieve the required data with considerably low access time. To maintain the performance of broadcast strategy, we introduce a technique to find the optimal broadcast channel by determining the optimum number of database items to be broadcast in a channel. The optimum number is used as an indication of when the broadcast cycle needs to be split into different channel, forming multiple channels.

We develop cost models to find the average access time of on-demand channel and broadcast channel. The cost models are applied with focusing on request that returns a single data item. To locate the optimum value of broadcast items, we compare the access time of broadcast channel and on-demand channel. The access time of broadcast channel is calculated based on certain factors, particularly number of database items to be broadcast, size of data item, and downlink bandwidth. As for on-demand channel, it takes into account the server service rate, request arrival rate, number of requests, size of request, uplink and downlink bandwidth. The optimum number of broadcast items is changed dynamically depending on the value of these factors. We also conduct a simulation to see the performance of our cost model in determining the optimum broadcast items. The simulation results show our cost models are considerably close to accurate.

For future work, we will consider multi data items retrieval in a single request, which includes a non-uniform size of request and data items. We will also measure the tuning time or the amount of time a client must listen to the channel, which is used to indicate its energy consumption. 


\section{References}

1. Acharya S., Alonso R., Franklin M. and Zdonik S., "Broadcast Disks: Data Management for Asymmetric Communication Environments", Proceedings of ACM Sigmod International Conference on Management of Data, pp.199-210, May, 1995.

2. Barbara D., "Mobile Computing and Databases - A Survey", IEEE Transactions on Knowledge and Data Engineering, Vol.11, No. 1, pp.108-117, January/February, 1999.

3. Badrinath B. R. and Phatak S. H., "An Architecture for Mobile Databases", Technical Report DCS-TR-351, Department of Computer Science, Rutgers University, New Jersey

4. Hu Q., Lee D. L. and Lee W.C., "Optimal Channel Allocation for Data Dissemination in Mobile Computing Environments", Proceedings of 18th International Conference on Distributed Computing Systems, pp.480-487, May, 1998.

5. Huang Y., Sistla P. and Wolfson O., "Data Replication for Mobile Computers", Proceedings of the ACM SIGMOD, pp.13-24, June, 1994.

6. Imielinski T. and Viswanathan S., "Adaptive Wireless Information Systems", Proceedings of SIGDBS (Special Interest Group in Database Systems) Conference, October, 1994.

7. Imielinski T., Viswanathan S. and Badrinath B. R., "Data on Air: Organisation and Access", IEEE Transactions on Knowledge and Data Engineering, Vol.9, No. 3, pp.353371, May/June, 1997.

8. Imielinski T., Viswanathan S. and Badrinath B. R., "Energy Efficient Indexing on Air", Proceedings of the ACM Sigmod Conference, pp.25-36, May, 1994.

9. Leong H. V. and Si A., "Database Caching Over the Air-Storage", The Computer Journal, Vol.40, No. 7, pp.401-415, 1997.

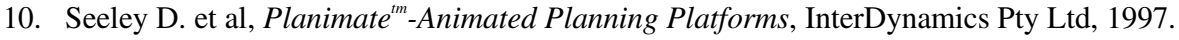

11. Sheng S., Chandrasekaran A. and Broderson R. W., "A Portable Multimedia Terminal for Personal Communication", IEEE Communications, pp.64-75, December, 1992.

12. Si A. and Leong H. V., "Query Optimization for Broadcast Database", Data and Knowledge Engineering, Vol.29, No. 3, pp.351-380, March, 1999.

13. Waluyo, A.B., Srinivasan B., Taniar D., "Current Trend in Mobile Database Query Optimization”, submitted to ACM Transactions on Database Systems, 2003. 